\title{
Goodness ratings of melodic openings and closures
}

\author{
PIET G. VOS and DENNIS PASVEER \\ University of Nijmegen, Nijmegen, The Netherlands
}

\begin{abstract}
The purpose of the present study was to map the suitability of melodic intervals for opening and closing a melody. Listeners $(n=13)$, belonging to two groups with different levels of expertise, rated the 25 within-octave intervals (unison plus the 12 nonunison intervals in ascending and in descending directions), for their suitability as openings; another, similarly subdivided group of listeners $(n=12)$ rated the intervals as candidates for closure. Both data sets were modeled by means of multiple regression. For openings, a three-factorial model (intervallic size, direction, and implicit harmony) provided a satisfactory description of the data; for closures, an extra factor (intervallic gravity) had to be added to the model. Differences involving level of expertise were established-notably, the importance of harmony for the experts, which played only a subordinate role in the responses of the nonexperts. The two sets of data were contrasted with those of closely related perceptual studies of openings and closures. In addition, the findings were discussed in relation to their contribution to insight into overlapping, a syntactical construction in which tones serve the double function of closing a musical phrase and opening the next one.
\end{abstract}

It is well known that the appreciation of a piece of music depends on numerous and heterogeneous factors that are virtually impossible to grasp altogether in one scientifically sound theory. Yet the issue is fascinating, given the fact that music is, like language, a universal tool for human communication. A parsimonious way to get insight into music processing is to select a smallest possible meaningful aspect of music for empirical research. Melodic intervals are such elementary and universal constituents of music. One could argue that single tones are still more elementary building stones for music, but musical structure is generated from relations between tones, and a (melodic) interval is but a special case of a relation between two immediately successive tones. Thus, an investigation of the perceptual aspects of intervals might also be informative about more complex aspects of music perception.

An interval is measured here in equally tempered semitones between two immediately successive tones. If we restrict ourselves to melodies that (could) belong to the domain of Western tonal music, disregarding intervals larger than an octave, because they are extremely rare in this domain (see Dowling, 1967; Dowling \& Harwood, 1986; Fucks, 1962; Vos \& Troost, 1989), and specifying an interval not only in terms of size ( $n$ semitones), but also in terms of direction (ascending vs. descending), 25 different possible intervals are available for the composition of a melody. These are the unison plus the 12 nonunison intervals, once

The authors thank Wolfgang Auhagen, Jorg Dautzenberg, Jules Ellis, Ab de Haan, Hank Heijink, Erik Jansen, and David Omtzigt for valuable advice, suggestions, and discussions during the preparation of the present study. Correspondence concerning this article should be addressed to P. G. Vos, NICI, University of Nijmegen, The Netherlands (e-mail: vos@nici.kun.nl). in the ascending direction (in the middle octave $\mathrm{C}_{4}-\mathrm{C}_{4}$, $\ldots, \mathrm{C}_{4}-\mathrm{C}_{5}$ ) and another time in the descending direction $\left(\mathrm{C}_{4}-\mathrm{C}_{4}, \ldots, \mathrm{C}_{5}-\mathrm{C}_{4}\right.$ ) (Table 1). Not all of them occur equally often in a melody that sounds good tonally. For example, small intervals, by and large, predominate over large ones in most melodies (Densmore, 1929; Dowling, 1967; Dowling \& Harwood, 1986; Meyer, 1956, 1973; Vos \& Troost, 1989; Watt, 1924; Zipf, 1949). The reason for this restriction on interval size is that it warrants melodic coherence (see Dowling, 1967; Heise \& Miller, 1951; van Noorden, 1975). Furthermore, small intervals, up to the fourth, statistically predominate in the descending direction, whereas larger intervals show the opposite pattern (Vos \& Troost, 1989). The constraints in question are compatible with Meyer's (1973) so-called bridge gap hypothesis, according to which, in order to arouse the listener's interest, a large ascending interval should occasionally be inserted into a melody, after which, in order to "restore" the melodic coherence, subsequent small intervals should be "given back" in the opposite - that is, descending - fashion. Only one notorious exception has been found to this rule: The fifth in (harmonized) melodies belonging to the repertoire of such divergent composers as J. S. Bach, Beethoven, Debussy, and Stravinsky occurs significantly more often in descending fashion (Vos \& Troost, 1989). The reason is most probably that a descending fifth means a natural, cadential return from a dominant to a tonic.

It is also intuitively clear that the composer's freedom in the use of intervals is dependent on whether they have to be used for an opening, a continuation/elaboration, or a closure of a melody. Since, in Western tonal music, a melody typically ends on the tonic, interval variation for the closure of a melody is more restricted than anywhere else in a 
Table 1

Survey of the 13 Within-Octave Intervals: Their Size in Semitones, the Ratio of the Within-Interval Tone Frequencies According to the Just Intonation, and Examples in Both Ascending and Descending Directions

\begin{tabular}{lccc}
\hline Melodic Interval & Semitones & Frequency Ratio & Example \\
\hline Unison & 0 & $1: 1$ & $\mathrm{C}_{4}-\mathrm{C}_{4}$ \\
Minor second & 1 & $16: 15$ & $\mathrm{C}_{4}-\mathrm{C}_{4} / \mathrm{C}_{4}-\mathrm{B}_{3}$ \\
Major second & 2 & $9: 8$ & $\mathrm{C}_{4}-\mathrm{D}_{4} / \mathrm{C}_{4}-\mathrm{A}_{3}$ \\
Minor third & 3 & $6: 5$ & $\mathrm{C}_{4}-\mathrm{D}_{4} / \mathrm{C}_{4}-\mathrm{A}_{3}$ \\
Major third & 4 & $5: 4$ & $\mathrm{C}_{4}-\mathrm{E}_{4} / \mathrm{C}_{4}-\mathrm{G}_{3}$ \\
Perfect fourth & 5 & $4: 3$ & $\mathrm{C}_{4}-\mathrm{F}_{4} / \mathrm{C}_{4}-\mathrm{G}_{3}$ \\
Tritone & 6 & $45: 32$ & $\mathrm{C}_{4}-\mathrm{F}_{4} \mathbb{H}_{4} / \mathrm{C}_{4}-\mathrm{F}_{3}$ \\
Perfect fifth & 7 & $3: 2$ & $\mathrm{C}_{4}-\mathrm{G}_{4} / \mathrm{C}_{4}-\mathrm{F}_{3}$ \\
Minor sixth & 8 & $8: 5$ & $\mathrm{C}_{4}-\mathrm{G}_{4} / \mathrm{C}_{4}-\mathrm{E}_{3}$ \\
Major sixth & 9 & $5: 3$ & $\mathrm{C}_{4}-\mathrm{G}_{4} / \mathrm{C}_{4}-\mathrm{D}_{3}$ \\
Minor seventh & 10 & $9: 5$ & $\mathrm{C}_{4}-\mathrm{A}_{4} / \mathrm{C}_{4}-\mathrm{D}$ \\
Major seventh & 11 & $15: 8$ & $\mathrm{C}_{4}-\mathrm{B}_{4} / \mathrm{C}_{4}-\mathrm{C}_{3}$ \\
Octave & 12 & $2: 1$ & $\mathrm{C}_{4}-\mathrm{C}_{5} / \mathrm{C}_{4}-\mathrm{C}_{3}$ \\
\hline
\end{tabular}

melody. Openings, to the contrary, seem to allow for much freedom of choice in this aspect, but here, too, there exist typical constraints, since a composer may be assumed to introduce quickly the major tonal intervals that characterize the melody's key - notably, the third and the dominant (see Vos, 1999). In relation to this point, it has recently been discovered that two intervals are privileged openings, in the sense that they firmly establish the key of a melody-namely, the ascending fourth (five semitones) and its tonal counterpart, the descending fifth (seven semitones). If a melody opens with one of these intervals, the second tone will almost always be the tonic, and consequently, the first one will be its dominant (Vos, 1999). The reason for this constraint is still unclear, but it may have to do with effecting an optimal priming of the melody's key in the listener. Ascending fourth openings are favorites particularly in national anthems and folksongs.

In the present study, our intention was to map the listener's evaluation of the 25 different possible intervals (Table 1) as candidates for the opening of a melody (Experiment 1) and for the closure of a melody (Experiment 2). Specifically, we wanted to test a simple theory that the listener's goodness judgments for each of the two melodic categories is a function of relevant stimulus factors-notably, (interval) size, direction, and harmony. Since it is well known that the listener's evaluation of musical stimuli is influenced by musical expertise, the impact of this factor is also examined in the experiments.

\section{EXPERIMENT 1}

\section{Intervals as Candidates for Melodic Openings}

As compared with the massive musicological literature on the closure (cadence) of a composition, studies of openings (incipits) are virtually nonexistent. Only in musicological treatises of previous centuries (e.g., in historical order, Zarlino, 1558/1997; Marpurg, 1753/1972; Kirnberger, 1776/1968; Weber, 1818; Sechter, 1854) does one find prescriptive suggestions on "good" openings, related to issues of the tonal goodness of compositions as a whole. For instance, it is written that the composer ought to open with consonant intervals and quickly come up with the harmonically most important intervals, such as the third, the fourth, and the fifth (see Vos, 1999). We were unable to find treatises dealing more technically with intervals as candidates for openings. A preliminary conclusion to be drawn from the poverty of the studies in question would be that musical openings are far less important than, for example, openings in chess games and that every interval might be an equally promising opening candidate. The reason for the musicological lack of interest is presumably the fact that cadences, rather than openings, form the keystone of the historical development of syntactical constraints in Western tonal music (see Anderson, 1992; Eberlein, 1994; Eberlein \& Fricke, 1992; Werts, 1983). It remains to be seen whether listeners are equally "indifferent" with respect to the plausibility of the different possible intervals as candidates for openings.

Turning now to the findings of music statistics on openings, prior to the above-mentioned discovery of the privileged status of ascending fourth and descending fifth openings, it had been established (Vos \& Troost, 1989) that a significant majority of several hundred openings, examined for the direction of intervals in incipits as a function of their size, consisted of ascenders. This fact is also indicated by the asymmetry of occurrence between ascending fourth and descending fifth openings: The latter constituted a very small minority in Vos's (1999) study. Taking these findings together and including the finding of the overall dominance of small intervals over large ones, we hypothesize that listeners will show similar patterns in the evaluation of the complete set of intervals as candidates for openings-namely, smaller ones will be better than larger ones, and ascenders will be better than their counterparts.

We now turn to another factor that might contribute to the listener's appreciation of the suitability of intervals for opening a melody-namely, harmony. This term is used with two different meanings, depending on whether it refers to simultaneously sounding tones, also called bichords, or to sequentially presented tones, as in melodic intervals. In the former sense of the term, one also speaks of explicit harmony, whereas it is convenient to speak of implicit (also called implied) harmony in the latter sense of the term (see, e.g., Holleran, Jones, \& Butler, 1995). The degree of (implicit) harmony of intervals is defined here in terms of both music-theoretical and music-historical facts about consonance, and not in psychological terms of perceived and/or evaluated consonance. Speaking in the terms of music theory, the degree of harmony of an interval corresponds with both its position in the series of harmonics and the simplicity of the frequency ratio between the two tones. The octave and its (octave) equivalent, the unison, are the most consonant intervals, followed by the fifth and its equivalent, the fourth. These so-called perfect consonant intervals also formed the his- 
torical basis of Western tonal music (Randel, 1986). Next in order are the major third and its equivalent, the minor sixth, followed by the minor-third/major-sixth. These four intervals are called imperfect consonants. Then come the major-second/minor-seventh, followed by the minorsecond/major-seventh and, finally, the tritone, the most dissonant of intervals, which does not occur at all in the series of harmonics.

A fourth factor may contribute to the appreciation of the goodness of an opening-namely, its metrical context. There are two possibilities: Either the first tone has, metrically speaking, an (accentuated) downbeat function, or it has a (nonaccentuated) upbeat function, preceding the proper downbeat. Recently (Vos, 1999), it has been established that, on average, in almost $70 \%$ of ascending fourth openings, the first tone was an upbeat. It suggests that an upbeat context will strengthen the opening character of a melodic interval. Therefore, it makes sense to examine the listener's evaluation of intervals as candidates for openings also as a function of metrical context.

\section{Method}

Subjects. Thirteen persons, staff members and students of the University of Nijmegen, participated in the experiment. All of them reported listening regularly to music belonging to the broadly defined repertoire of Western tonal music. Six of them were musically trained, in the sense that they had taken an instrument course for at least 2 years and were still involved in making music. The age of the subjects varied between 20 and 31 years (median age, 26 years). Participation was paid for by curriculum credit points or a modest amount of money.

Stimulus materials. Each stimulus consisted of two sequentially presented tones, generated by a soundcard (Creative SB Live!). The tones (timbre, "bright piano") and the rests between tones lasted $290 \mathrm{msec}$ each, shaping a moderato tempo. The pitches of the tones (equal-tempered tuning, $\mathrm{A}_{4}=440 \mathrm{~Hz}$ ) ranged between $\mathrm{F}_{3}$ $(185 \mathrm{~Hz})$ and $\mathrm{F}_{5}(698 \mathrm{~Hz})$. The pitch of the first tone was selected pseudorandomly, in the sense that the second tone had to fall within the just-specif ied range. Consequently, the majority of the first tones stemmed from the central part of the range, whereas the second tones were equally distributed over the full range. Loudness was fixed at a level of comfort estimated a priori for the listeners. The 25 stimuli (the unison plus the 12 nonunison intervals in both directions) were embedded in two different metric contexts, either upbeat or downbeat. In the upbeat variant, the stimulus was accompanied by a weakly sounding sequence of five (metronome) clicks, three of which preceded the tones of the stimulus; in the downbeat variant, a similarly shaped sequence, now consisting of six clicks, accompanied the stimulus, four of which preceded the tones of the stimulus. In both contexts, the first and the fifth metronome clicks were played louder than the others in order to create either an upbeat or a downbeat accentuation within a 4/4 metric framework (Figure 1).
Procedu re. The subject, wearing headphones, was seated in front of a PC-controlled monitor. The instructions, presented on the screen, informed the subject that the intention of the experiment was to map the listener's evaluation of the goodness of the openings of melodies, constrained to the first two tones of an arbitrary melody. In addition, the subject was told that each stimulus (melodic interval) was embedded in a metric context suggesting either a downbeat or an upbeat interpretation of the stimulus. After stimulus presentation, the subject indicated the evaluative response by moving a pointer, initially positioned at the center of a sliding scale, off the center position according to the following scale specification: Moving the pointer to the left would signify an increasingly bad opening; moving the pointer to the right would mean an increasingly good opening. In the data analysis, the range of responses was numerically coded as ranging from 0 to 100 . After the subject had responded, the pointer was automatically reset to the center of the scale, after which the subject could either repeat the trial once again or initiate the next trial. The subject was encouraged to use the full range of the scale.

The pitch of the first tone of a stimulus was determined in a pseudorandom manner that prevented the first tones in two immediately successive trials from having had the same pitch. The pseudorandomization was also arranged in such a way as to prevent the immediate succession of more than three similarly sized or similarly oriented intervals.

Five exercise stimuli (one unison and the others nonunison; three in downbeat fashion and two in upbeat fashion) served to make the subject familiar with both the sound and the shape of the stimuli and with the experimental procedure.

The two blocks of 50 stimuli each were presented in an uninterrupted order. It took the subjects, on average, approximately $30 \mathrm{~min}$ to complete the experiment.

\section{Results}

The complete set of 1,300 data was subjected to a multiple linear regression analysis. The factors were size, direction, harmony, and meter. Size was expressed in terms of semitones, from 0 (unison) to 12 (octave), with the hypothesis being that goodness would be negatively related to interval size. Direction was coded as 0 (unison), -1 (descending direction), or 1 (ascending direction), with the hypothesis being that ascending intervals would be evaluated as more favorable than descending intervals. Harmony was coded as follows: unison $=0$; tritone $=1$; minorsixth/major-seventh $=2$; major-second/minor-seventh $=$ 3 ; minor-third/major-sixth $=4$; major-third/minor-sixth $=$ 5 ; perfect-fourth/perfect-fifth $=6$; octave $=7$. The reason for coding the unison as 0 was that, because of its single pitch, this interval has no harmonic valence in openings or endings but primarily serves rhythmic purposes. It was hypothesized that goodness would be positively related to harmony. The factor of beat was binary coded as 0 for downbeat and as 1 for upbeat, with the hypothesis being that stimuli presented in an upbeat context
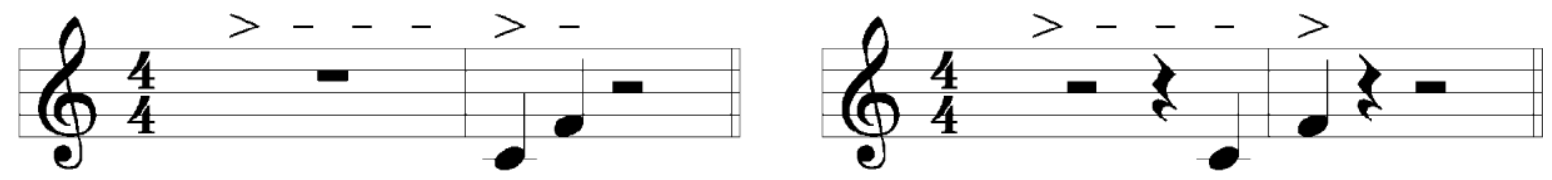

Figure 1. Schematic illustration of the downbeat (left) and the upbeat (right) accompaniments of the stimuli. 
would be rated as better openings than were stimuli presented in a downbeat context.

First, the impact of the factor of beat was examined. For the experts, $\beta$ was $.000, p=.997$; for the nonexperts, $\beta$ was $-.041, p=.215$. Hence, it is concluded that beat has no differential effect on the goodness ratings of openings. Inspection of the data for the various intervals showed that even the predictions for the two potentially highest ranking candidates were not confirmed, as is illustrated here by following the experts' data: ascending fourth, $d$ (the mean rating difference between the two conditions) $=5.02$, $t(22)=-0.584, p=.565$; descending fifth, $d=11.61$, $t(22)=-1.123, p=.274$. Consequently, the data for the separate beat conditions were combined into one set of data, and a three-factorial model (size, direction, and harmony) was tested for each expert group separately.

Experts. Examination of the (standardized) $\beta$ coefficients proved that all three of them were highly significant $(p<.001)$. The coefficients were -.425 for size, .127 for direction, and .473 for harmony. Note that the sign of each coefficient was in agreement with the corresponding hypothesis. Figure $2 \mathrm{~A}$ shows, for each of the 25 intervals, the data means and the predictions.

The goodness of fit between the data and the predictions, as is shown in Figure 2A, was calculated in terms of the percentage of explained variance, using the formula $R^{2}=$
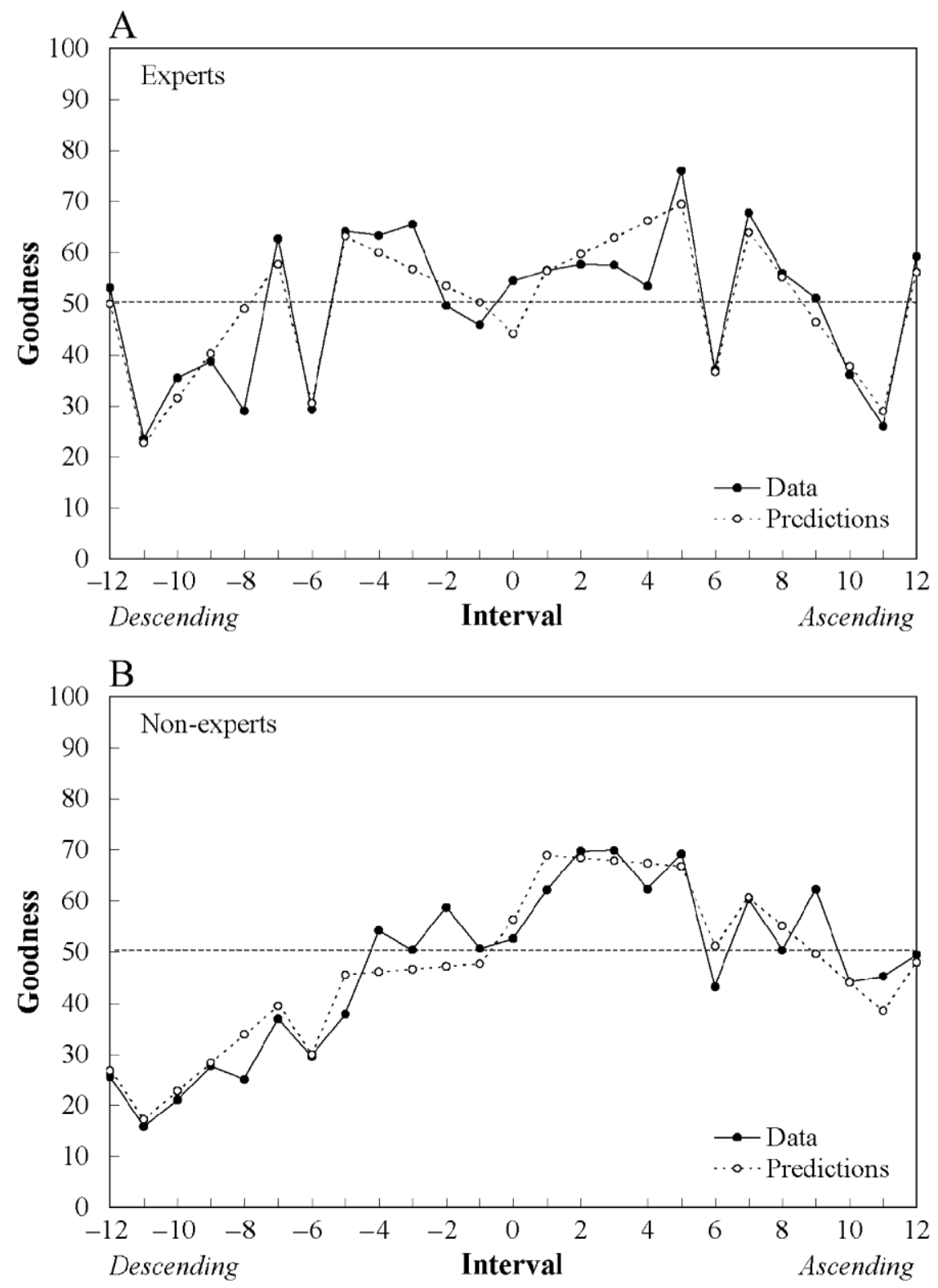

Figure 2. Goodness ratings for the 25 intervals. (A) Mean expert ratings; (B) mean nonexpert ratings. See Table 1 for the names of the intervals listed on the $\boldsymbol{x}$-axis. 
1 - (prediction variance)/(data variance). $R^{2}$ was .816 . By using the $t$ test, significantly incorrect predictions were established for (1) the descending minor sixth $[d=18.75$, $t(23)=-4.780, p<.001],(2)$ the ascending major third $[d=12.14, t(23)=-2.611, p=.016]$, and (3) the unison $[d=-10.78, t(23)=2.908, p=.008]$. It is noted that the first two intervals, rated higher than was predicted, are octave equivalent. We will come back to these and subsequent incorrect predictions in the General Discussion section.

Nonexperts. All three $\beta$ coefficients were highly significant $(p<.001)$. The values were -.373 for size, .353 for direction, and .145 for harmony. The signs of the coefficients were in agreement with the corresponding hypotheses. Figure 2B shows, for each of the 25 intervals, the data means and the predictions. $R^{2}$ was .883 . Application of the $t$ test showed that one prediction was significantly incorrect-namely, that for the ascending major sixth $[d=-12.67, t(27)=3.245, p=.003]$.

The correlation between the data for the two groups (Pearson correlation based on mean responses for each interval) was $R=.641, p=.001$. Comparing the data of the experts with those of the nonexperts, it is seen that, for the experts, size and harmony were most important, whereas the nonexperts primarily based their goodness ratings on the size and direction of the intervals. The contrast in the appreciation of harmony is nicely exemplified in the experts' rating peaks for ascending fourths and descending fifths and their rating dips for tritones, which are hardly salient in the rating pattern of the nonexperts. The fact that direction was more important for the nonexperts than for the experts is demonstrated in the shape of the nonex- perts' curve, in that the left part is low and the right one is high. In addition, the ratings of the nonexperts were more flattened throughout, a fact that had also been reported to be a distinctive feature of nonexperts in other experimental studies of music perception (e.g. Cuddy, Cohen, \& Mewhort, 1981; Cuddy \& Lunney, 1995; Vos \& Verkaart, 1999).

Although globally the predictions followed the data, there is one apparent contradiction in the experts' data: the unexpectedly low rating for the descending minor sixth, which is tonally equivalent to the ascending major third. We have no explanation for this violation of the experts' higher sensitivity for harmony.

One possible explanation for the lack of significance of the factor of beat is that the difference between up- and downbeat becomes effective only with larger segments of melodies. Another inhibitory factor might have been the insufficient degree of salience with which the accentuation was realized in the present experiment. We will examine further conclusions and points for discussion in the General Discussion section, where we will compare the present results with the outcomes for the goodness ratings for closures.

\section{EXPERIMENT 2 \\ Intervals as Candidates for Melodic Closures}

In the introduction, it has already been emphasized that closures have considerably fewer degrees of freedom than do openings. In fact, almost always, a piece of tonal music ends with a cadence toward a closure endowed with tonic harmony (see, e.g., the lemma on "Cadence" in Randel, 1986). Among musicians, it is often said that, if one wants

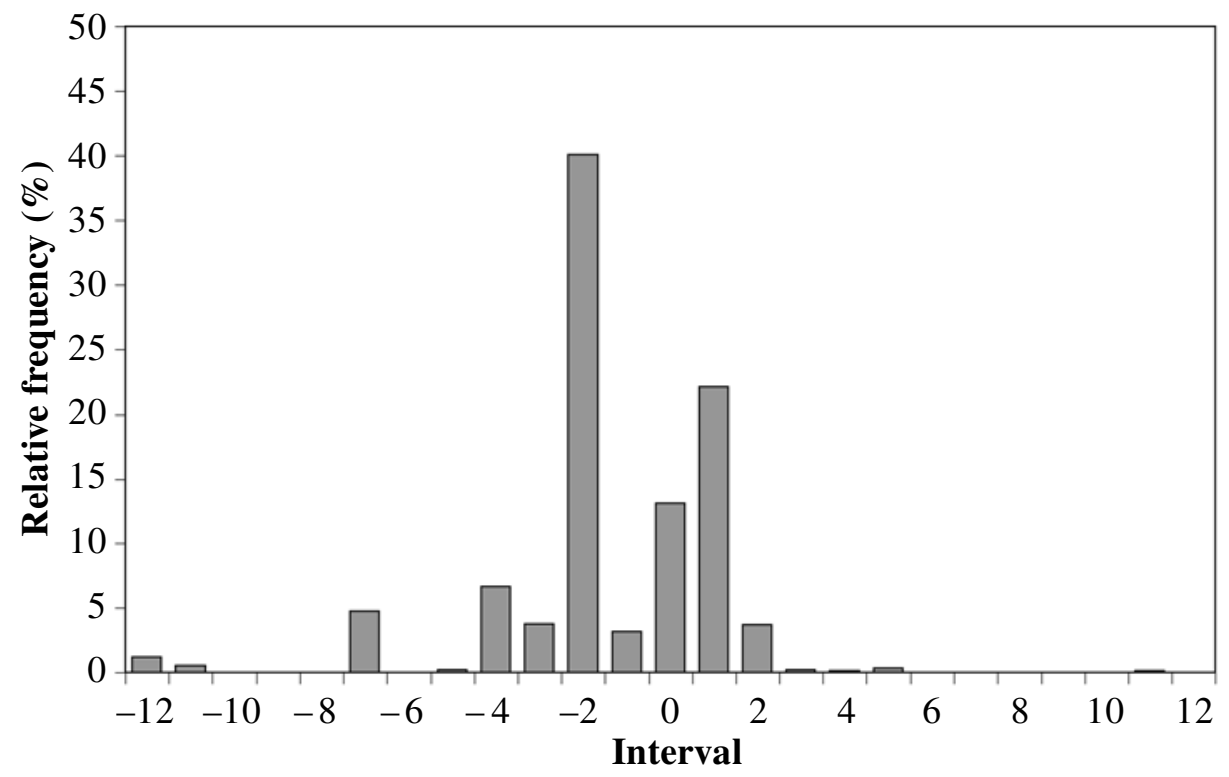

Figure 3. Frequency distribution of the 25 intervals used to close existing $(N=941)$ melodies, arbitrarily selected from the repertoires of baroque, classical, and romantic melodies, and from West European folksongs. See Table 1 for the names of the intervals listed on the $x$-axis. 


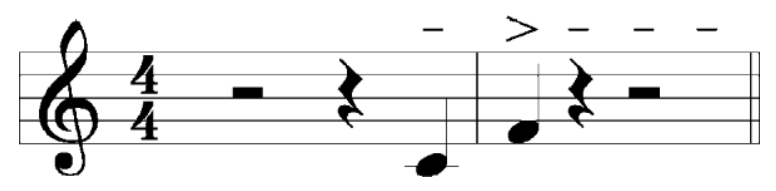

Figure 4. Schematic illustration of the beat accompaniments of the stimuli.

to know the key in which a piece of music has been written, one can, as a rule of thumb, infer it from the last note or chord. The fact that the last note of a melody is conventionally the tonic also imposes restrictions on the selection of the tone prior to the last tone. In other words, the repertoire of melodic intervals that can be used for the closure of a melody will also be limited. This can easily be verified by an examination of the closures of existing pieces of music, normalized to the key of C (Figure 3). The majority of them consist of descending major seconds and ascending minor seconds. The reason is that, in the key of $\mathrm{C}, \mathrm{D}-\mathrm{C}$ and $\mathrm{B}-\mathrm{C}$ are stepwise returns to the tonic, $\mathrm{D}-\mathrm{C}$ in the descending and $\mathrm{B}-\mathrm{C}$ in the ascending direction. In addition, $\mathrm{B}$ is the leading tone to a tonic (see the lemmas on "leading tone" and "stepwise" in Randel, 1986). Therefore, to predict listeners' goodness ratings of intervals as closure candidates, it does not seem sufficient to use the three-factorial model used for openings. Although those three factors remain meaningful, they do not completely account for the strength with which an interval functionally implies a tendency toward the tonic. Beyond the privileged closure status of the two leading-tone intervals, we have to remember from the (general) introduction two other intervals with similar tonal implications-namely, the ascending fourth and the descending fifth.

The question arises, then, as to whether there exists a more general rule that determines the likelihood that any interval will or will not be endowed with the same functional tonal implication as the four just-mentioned intervals. Such a rule is found in the so-called Lipps's law (Lipps, 1901; see Van Dyke Bingham, 1910, for an application of Lipps's law in an experimental investigation of the perception of melodic intervals, and Weinmann, 1904, for an investigation of the theoretical implications of Lipps's law for understanding the structure of tonal melodies). ${ }^{1}$ Briefly formulated, the law states that if two tones composing a melodic interval are related as $f_{1}: f_{2}=p: q=2^{n}: 3,5,7, \ldots$, tone $p$ has the functional implication that it will become the interval's center of gravity, meaning that the other tone of the interval is perceptually attracted to that tone. From this law, or as it would be more modestly called nowadays, rule, one can derive which of the 25 intervals in our investigation will be perceptually plausible closure candidates. When the second tone of a melodic interval functions as a center of gravity, in the sense of Lipps's law, tonally it becomes a candidate to be the tonic of the interval. In the absence of any further melodic context, we hypothesize that intervals ending on such a center of gravity are more likely to be perceived as a closure than are other intervals.
Consequently, we expanded the three-factorial model used for openings into a four-factorial one, with the fourth factor, gravity, coded as follows. Intervals in which the second tone has the functional implication of being the center of gravity $\left(p=2^{n}\right)$, were coded as 1 ; other intervals were coded as 0 . Hence, the ascending minor second $\left(2^{4}: 15\right)$, perfect fourth $\left(2^{2}: 3\right)$, and minor sixth $\left(2^{3}: 5\right)$ and the descending major second $\left(2^{3}: 9\right)$, major third $\left(2^{2}: 5\right)$, tritone $\left(2^{5}: 45\right)$, perfect fifth $\left(2^{1}: 3\right)$, and major seventh $\left(2^{3}: 15\right)$ were coded as 1 . All the other intervals were coded as 0 .

\section{Method}

Subjects. Twelve persons took part in the experiment. They were recruited from among staff members and students of the University of Nijmegen and had not participated in Experiment 1. All of them were familiar with current Western tonal music. Five of them were experts according to the criteria also used for the selection of experts in Experiment 1. The 7 nonexperts had a musical background similar to that of the nonexperts in Experiment 1.

Stimulus materials. The stimuli were exactly the same as those used in Experiment 1. The metrical context consisted of six beats, the first two of which coincided with the tones of the stimulus, with the accentuated beat on the second tone (Figure 4).

Procedure. The procedure was exactly the same as the one used in Experiment 1, except for the following details. In the instructions, the term opening was replaced by closure, and a similar substitution was introduced for the meaning of adjustment responses (moving the pointer to the right away from the starting position indicated, increasingly, good closure). The beat context differed as specified above. Finally, the complete set of 25 stimuli was presented four times, divided into two blocks of $2 \times 25$ trials, with a short break between the two blocks.

\section{Results}

The set of 1,200 data was subjected to a multiple regression analysis with the factors of size, direction, harmony, and gravity, as specified above, and split out per expertise group.

Experts. The $\beta$ coefficients proved to be significant only for size $(-.236, p<.001)$, harmony $(.255, p<.001)$, and gravity $(.273, p<.001)$. For direction, $\beta$ was -.037 , $p=.373$. In Figure 5A, the data means and the predictions are shown for each of the 25 intervals. Goodness of fit between the data and the predictions was established by the computation of $R^{2}$, which equaled .489. Application of the $t$ test showed that 10 predictions were incorrect, among which, notably, were those for the ascending minor second $[d=-20.16, t(19)=3.493, p=.002]$, major third $[d=14.12, t(19)=-2.387, p=.028]$ and octave $[d=-21.86, t(19)=2.533, p=.020]$ and for the descending minor sixth $[d=13.80, t(19)=-2.472, p<$ $.001]$ and octave $[d=-34.62, t(19)=5.201, p<.001]$. It should be noted that the predictions were also positive for the ascending major third and the descending minor sixth, as was found similarly in the experiment with openings, whereas those for the other specified intervals were clearly too negative.

Nonexperts. The $\beta$ coefficients proved to be significant for the factors of size $(-.081, p<.001)$, direction $(-.340, p<.001)$, and gravity $(.137, p<.001)$, but not for harmony $(.050, p=.180)$. In Figure $5 \mathrm{~B}$, the data 

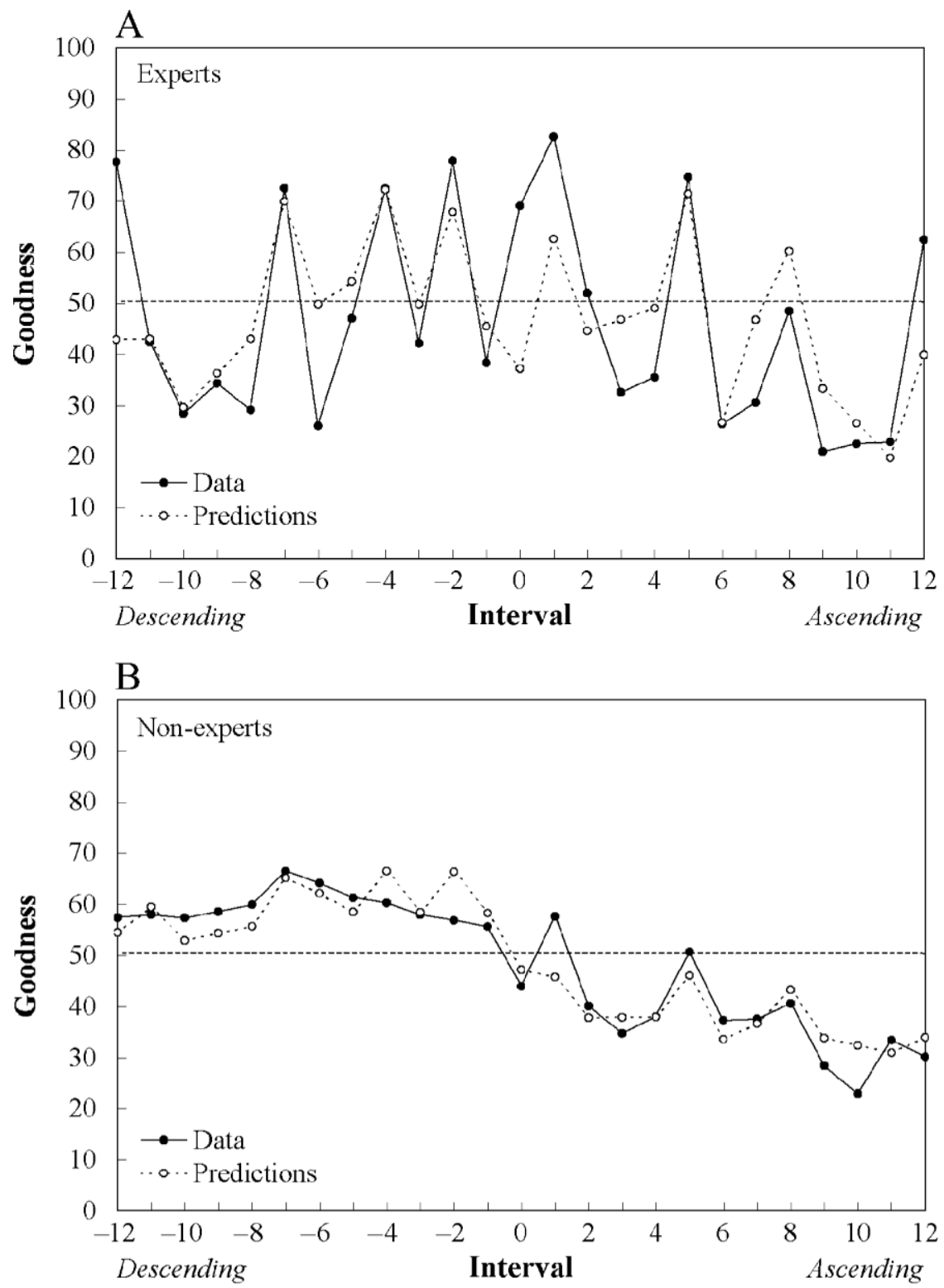

Figure 5. Goodness ratings for the 25 intervals. (A) Mean expert ratings; (B) mean nonexpert ratings. See Table 1 for the names of the intervals listed on the $x$ - axis.

means and predictions are shown as a function of interval. Goodness of fit, calculated in terms of $R^{2}$, was .858 .

Incorrect predictions were restricted to only one interval, the ascending minor seventh $[d=9.23, t(27)=$ $-2.355, p=.026]$. The correlation between the two groups, calculated in the same manner as for the data for the experiment with openings, was only marginally significant $(R=.372, p=.067)$. Comparing the data from the two expertise groups, one can observe similar differences to those established in the experiment with openings. Thus, nonexperts' ratings were more flattened. Again, harmony appeared to be important for the experts, whereas this factor played, at most, a minor role for the nonexperts. Whereas direction did not matter for the experts, it was most important for the nonexperts. Finally, the gravity factor proved to be honored by both expertise groups.

\section{GENERAL DISCUSSION}

The present study has mapped the suitability of the different within-octave intervals for carrying two important functions in music-namely, to open and to close a piece of music. It appeared to be possible to predict the goodness ratings for both functions in a rather satisfactory way on the basis of an interplay among only a few elemen- 
tary interval attributes. At the same time, it was established that the weight of these distinct attributes systematically differed as a function of the level of musical expertise among listeners. Specifically, the harmony of intervals was throughout more important for expert listeners, both for openings and for closures. This latter finding is not really surprising, given the fact that experienced musicians dispose of a broad knowledge of tonal - that is, both melodic and harmonic-contexts in which an isolated interval might be embedded (see Auhagen \& Vos, 2000). Nonexperts, on the other hand, are particularly sensitive to intervallic direction.

It is worthwhile to know which intervals scored comparably high (or low) for both musical functions. Since the judgments of the experts seem to be more valid than those of the nonexperts, we here consider only the experts' ratings (Figures 2A and 5A). Notoriously bad candidates appeared to be the tritone, as well as the major and minor seventh in both directions. Among the good candidates are the ascending fourth, its tonal equivalent, the descending fifth, as well as the descending major third.

It is relevant to contrast the present study with two closely related ones, one of which (Cuddy \& Lunney, 1995) bears similarity with our investigation of openings, whereas the other one (Van Dyke Bingham, 1910) is similarly related to our investigation of closures. We start with the Cuddy and Lunney study of melodic continuation ratings. Briefly summarized, the authors examined listeners' goodness-of-continuation ratings for stimuli consisting of small (major second and minor third) and large (major sixth and minor seventh) intervals, in both ascending and descending directions, each of them, eight in all, followed by a third tone selected from the range of \pm 12 semitones, reckoned from the second tone. A considerably more complex linear multiple regression model (seven factors, including a number of principles from Narmour's, 1990, model of melodic expectancy, modified ad hoc) was tested, with satisfactory statistical results rather similar to those obtained for our model for openings. The step from a one-interval stimulus to a two-interval stimulus implies a considerable increase in melodic degrees of freedomhence, in complexity - both theoretically, as elaborated in the authors' model of the data, and in terms of the uncertainty as to exactly what quantitative manner a listener will use to weight the impact of even three intervals (including the interval between the first and the third tone) as a basis for goodness ratings. Under the assumption that the Cuddy and Lunney data for the stimulus conditions in which the second interval was unison (e.g. $E_{4}-G_{4}-G_{4}$ ) were comparable to our data for the same nonunison interval (here, $\mathrm{E}_{4}-\mathrm{G}_{4}$ ), we rescaled the Cuddy and Lunney data-rankings on a 7-point scale, with $7=$ very good continuation - to percentages. The eight continuation ratings were throughout higher and, moreover, positive for the large intervals (rated low in our experiment). However, the relative ranks, such as that for the descending minor seventh or compared with that for the major sixth in either direction, were largely similar to ours. Again, the explosive complexity with increase of stimulus length makes it difficult, if not impossible, to use our data for openings for predictions of goodness ratings for progressively longer melodic segments. It is worthwhile to note that, in a similar experiment, as reported in Cuddy and Lunney's study (Auhagen, 1994), listeners' continuation responses were strongly influenced by the V-I cadential scheme, a finding that is in accordance with our results.

Next, we compare our closure data with the data from the rather old but experimentally strong Van Dyke Bingham (1910) study of finality judgments of intervals. The major purpose of the author was to experimentally test Lipps's law for the tonal implication of intervals. The stimuli were the same as ours, except for the deletion of unison and octaves and the inclusion of the dimished fifth $\left(45: 2^{6}\right)$. The task of the subjects was to indicate whether a stimulus sounded as a melodic closure ("Can you make this second a final tone? Does this melody end?" p. 23). Responses consisted of yes, no, or ? The following intervals were evaluated positively, in the sense that the sum scores of yes, expressed as a proportion, were higher than .50: descending fifth, major third, and major second; ascending minor second, fourth, and minor sixth. The failure of the predictions of Lipps's law for the ascending minor seventh and descending major seventh were attributed to their "lack of relationship," a feature largely covered, in both Cuddy and Lunney's (1995) model and ours, by the factor of size ( proximity in Cuddy and Lunney's model). We may conclude, then, that Van Dyke Bingham's data were very similar to ours, except for the ascending major second, negatively evaluated but slightly positively rated in our experiment, and the ascending minor sixth, evaluated marginally positive by the subjects in Van Dyke Bingham's experiment and, contrary to our prediction, rated slightly negative in our experiment.

Although we addressed the goodness of intervals for opening and closing a piece of music, our data may perhaps be generalized to the appreciation of openings and closures of subsequent phrases within a musical composition. Now it should be noted that musical phrases can be linked by a unique syntactic construction that is forbidden in the case of (linguistic) sentence production: overlapping (in German, Verschraenkung; see Lerdahl \& Jackendoff, 1983). Overlapping means that a tone or several immediately successive tones both terminate a particular phrase and open the next one. Curiously, this syntactical construction has hardly been studied in a systematic manner. One reason for this neglect is its incompatibility with parsing models for musical phrases (e.g., Lehrdal \& Jackendoff, 1983; Tenney \& Polansky, 1980). However, the ubiquity of overlapping constructions suggests that composers apply it explicitly to "feed" the aesthetic surprise in the listener. Overlapping is, in fact, a special form of purposeful ambiguity which occurs also in various other forms in music (see Bernstein, 1976, and Thomson, 1983, for the role of ambiguity in the perception and appreciation of musical structure). Our data suggest that overlappings in the form of the ascending fourth, the descending 
major third, and the descending fifth have a better chance of being appreciated by the listener. The musicological reason for the appropriateness of those intervals for overlapping constructions is probably related to the fact that the second tone of these intervals has the functional implication of serving as a tonic (cf. gravity factor). A study of the occurrence of such overlaps in existing music, as well as of their appreciation by a listener, is clearly beyond the scope of the present study.

\section{REFERENCES}

ANDERSON, N. D. (1992). Aspects of early major-minor tonality. Unpublished doctoral dissertation, Ohio State University. (Available as http://web.presby.edu/ danderso/diss)

AuHAGen, W. (1994). Experimentelle Untersuchungen zur audiotiven Tonalitätsbestimmung in Melodien [Experimental investigations of auditory inference of tonality in melodies]. Kassel: G. Bosse.

Auhagen, W., \& Vos, P. G. (2000). Experimental methods in tonality induction research: A review. Music Perception, 17, 417-436.

Bernstein, L. (1976). The unanswered question. Cambridge, MA: Harvard University Press.

Cuddy, L. L., Cohen, A. J., \& Mewhort, D. J. K. (1981). Perception of structure in short melodic sequences. Journal of Experimental Psychology: Human Perception \& Performance, 7, 869-883.

Cuddy, L. L., \& LunNey, C. A. (1995). Expectancies generated by melodic intervals: Perceptual judgments of melodic continuity. Perception \& Psychophysics, 57, 451-462.

Densmore, F. (1929). Pawnee music. Washington, DC: Smithsonian Institution, Bureau of American Ethnology.

Dowling, W. J. (1967). Rhythmic fission and the perceptual organization of tone sequences. Unpublished doctoral dissertation, Harvard University.

Dowling, W. J., \& Harwood, D. L. (1986). Music cognition. New York: Academic Press.

EBERLEIN, R. (1994). Die Entstehung der tonalen Klangsyntax [The origin of tonal-harmonic syntax]. Kassel: Bärenreiter.

EBERLEIN, R, \& FRICKe, J. (1992). Kadenzwahrnehmung und Kadenzgeschichte [Perception and history of cadences]. Frankfurt: P. Lang.

FucKs, W. (1962). Mathematical analysis of formal structure of music. IRE Transactions on Information Theory, 8, 225-228.

Heise, G. A., \& Miller, G. A. (1951). An experimental study of auditory patterns. American Journal of Psychology, 64, 68-77.

Holleran, S., Jones, M. R., \& Butler, D. (1995). Perceiving implied harmony: The influence of melodic and harmonic context. Journal of Experimental Psychology: Learning, Memory, \& Cognition, 21, 737753.

KIRNBERGER,J. P. (1968). Die Kunst des reinen Satzes in der Musik [The art of well-formedness in music]. Hildesheim: G. Olms. (Original work published 1776)

Krumhansl, C. (1990). Cognitive foundations of musical pitch. Oxford: Oxford University Press.

LERDAHL, F., \& JACKENDOFF, R. (1983). A generative theory of tonal music. Cambridge, MA: MIT Press.

LIPPS, T. (1901). Zur Theorie der Melodie [On the theory of melody]. Zeitschrift für Psychologie und Physiologie der Sinnesorgane, 27, 225-263.

MARPURG, F. W. (1753). Analysen von Bachschen Fugenthemen, Fugen und Kanons [Analyses of fugue themes, fugues and canons by J. S. Bach]. In H.-J. Schulze, Bach Dokumente: Vol. 3. Dokumente zum Nachwirken Johann Sebastian Bachs 1750-1800. Leipzig and Kassel: Barenreiter. (Original work published 1753).

MeYer, L. (1956). Meaning and emotion in music. Chicago: University of Chicago Press.

Meyer, L. (1973). Explaining music: Essays and explorations. Chicago: University of Chicago Press.

NARMOUR, E. (1990). The analysis and cognition of basic melodic structures: The implication-realization model. Chicago: University of Chicago Press.

Povel, D.-J. (1996). Exploring the harmonic elementary forces in the tonal system. Psychological Research, 58, 274-283.

Randel, D. M. (1986). The new Harvard dictionary of music. Cambridge, MA: Harvard University Press, Belknap Press.

Sechter, S. (1854). Die Grundsätze der musikalischen Komposition [Fundamentals of musical composition]. Leipzig: Breitkopf und Härtel.

Tenney, J., \& Polansky, L. (1980). Temporal Gestalt perception in music. Journal of Music Theory, 24, 205-241.

Thomson, W. (1983). Functional ambiguity in musical structures. Music Perception, 1, 3-27.

Van Dy ke Bingham, W. (1910). Studies in melody. Psychological Review, 12 (Monograph Suppl., Whole No. 50).

VAN NoORdEN, L. P. A. S. (1975). Temporal coherence in the perception of tone sequences. Unpublished doctoral dissertation, Institute of Perception Research, Eindhoven.

Vos, P. G. (1999). Key implications of ascending fourth and descending fifth openings. Psychology of Music, 27, 4-18.

Vos, P. G., \& Troost, J. (1989). Ascending and descending melodic intervals: Statistical findings and their perceptual relevance. Music Perception, 6, 383-396.

Vos, P. G., \& VerkaArt, P. P. (1999). Inference of mode in melodies. Music Perception, 17, 223-239.

WatT, H. (1924). Function of the size of interval in the songs of Schubert and of the Chippewa and Sioux Indians. British Journal of Psychology, 14, 370-386.

Weber, G. (1818). Versuch einer geordneten Theorie der Tonkunst [Towards a systematic theory of musical composition]. Mainz: B. Schott.

Weinmann, F. (1904). Zur Struktur der Melodie [On the structure of melody]. Zeitschrift für Psychologie, 35, 340-379 and 410-453.

WERTS, D. (1983). A theory of scale references. Unpublished doctoral dissertation, Princeton University.

Zarlino, G. (1997). Musical treatises (Facsimile and transcription edited by F. Wiering. Thesaurus musicarum italicarum, Vol. I). (Original works published 1558-1589).

ZIPF, G. K. (1949). Human behavior and the principle of least effort. New York: Hafner.

\section{NOTE}

1. It should be noted that Lipps's theory of musical tonality has been proved to be problematic in various aspects of musical structure beyond the elementary level of the functional tonal implications of single intervals (see Auhagen, 1994, for a critical review of Lipps's theory, and Krumhansl, 1990, and Povel, 1996, for other recent reviews of Lipps's pioneering contributions to the psychology of music).

(Manuscript received January 23, 2001; revision accepted for publication August 16, 2001.) 